\title{
Nonlinear Degenerate Anisotropic Elliptic Equations with Variable Exponents and $L^{1}$ Data
}

\author{
KHELIFI Hichem ${ }^{1,2, *}$ and MOKHTARI Fares ${ }^{1}$ \\ ${ }^{1}$ Department of Mathematics and Informatics, University of Algiers, Algiers, \\ Algeria. 2 Street Didouche Mourad Algiers. \\ 2 Applied Mathematics Laboratory, Badji Mokhtar University-Annaba B.P. 12, \\ Algeria.
}

Received 31 March 2019; Accepted 24 January 2020

\begin{abstract}
This paper is devoted to the study of a nonlinear anisotropic elliptic equation with degenerate coercivity, lower order term and $L^{1}$ datum in appropriate anisotropic variable exponents Sobolev spaces. We obtain the existence of distributional solutions.
\end{abstract}

AMS Subject Classifications: 35J70, 35J60, 35D30

Chinese Library Classifications: $\mathrm{O} 175.27$

Key Words: Sobolev spaces with variable exponents; anisotropic equations; elliptic equations; $L^{1}$ data.

\section{Introduction}

In this paper we prove the existence of solutions to the nonlinear anisotropic degenerate elliptic equations with variable exponents, of the type

$$
\begin{array}{ll}
-\sum_{i=1}^{N} D_{i} a_{i}(x, u, \nabla u)+g(x, u, \nabla u)=f, & \text { in } \Omega, \\
u=0, & \text { on } \partial \Omega,
\end{array}
$$

where $\Omega \subseteq \mathbb{R}^{N}(N \geq 3)$ is a bounded domain with smooth boundary $\partial \Omega$ and the righthand side $f$ in $L^{1}(\Omega), D_{i} u=\frac{\partial u}{\partial x_{i}}$. We suppose that $a_{i}: \Omega \times \mathbb{R} \times \mathbb{R}^{N} \rightarrow \mathbb{R}, i=1, \ldots, N$ are

${ }^{*}$ Corresponding author. Email addresses: khelifi .hichemedp@gmail .com (H. Khelifi), f.mokhtari@univ-alg -er.dz (F. Mokhtari) 
Carathéodory functions such that for almost every $x$ in $\Omega$ and for every $(\sigma, \xi) \in \mathbb{R} \times \mathbb{R}^{N}$ the following assumptions are satisfied for all $i=1, \ldots, N$

$$
\begin{aligned}
& \left|a_{i}(x, \sigma, \xi)\right| \leq \beta\left(|k(x)|+|\sigma|^{\bar{p}(x)}+\sum_{j=1}^{N}\left|\xi_{j}\right|^{p_{j}(x)}\right)^{1-\frac{1}{p_{i}(x)}}, \\
& \sum_{i=1}^{N}\left(a_{i}(x, \sigma, \xi)-a_{i}(x, \sigma, \eta)\right)\left(\xi_{i}-\eta_{i}\right)>0, \quad \forall \xi \neq \eta, \\
& \sum_{i=1}^{N} a_{i}(x, \sigma, \xi) \xi_{i} \geq \alpha \sum_{i=1}^{N} \frac{\left|\xi_{i}\right|^{p_{i}(x)}}{(1+|\sigma|)^{\gamma_{i}(x)}},
\end{aligned}
$$

where $\beta>0, \alpha>0$, and $k \in L^{1}(\Omega), \gamma_{i}: \bar{\Omega} \rightarrow \mathbb{R}^{+}, p_{i}: \bar{\Omega} \rightarrow(1,+\infty)$ are continuous functions and $\bar{p}$ is such that

$$
\frac{1}{\bar{p}(\cdot)}=\frac{1}{N} \sum_{i=1}^{N} \frac{1}{p_{i}(\cdot)}
$$

We introduce the function

$$
\bar{p}^{*}(x)= \begin{cases}\frac{N \bar{p}(x)}{N-\bar{p}(x)}, & \text { if } \bar{p}(x)<N \\ +\infty, & \text { if } \bar{p}(x) \geq N .\end{cases}
$$

The nonlinear term $g: \Omega \times \mathbb{R} \times \mathbb{R}^{N} \rightarrow \mathbb{R}$ is a Carathéodory function such that for a.e. $x \in \Omega$ and all $(\sigma, \xi) \in \mathbb{R} \times \mathbb{R}^{N}$, we have

$$
\begin{aligned}
& |g(x, \sigma, \xi)| \leq b(|\sigma|)\left(c(x)+\sum_{i=1}^{N}\left|\xi_{i}\right|^{p_{i}(x)}\right), \\
& g(x, \sigma, \xi) \cdot \sigma \geq 0,
\end{aligned}
$$

where $b: \mathbb{R}^{+} \rightarrow \mathbb{R}^{+}$is a continuous and increasing function with finite values, $c \in L^{1}(\Omega)$ and $\exists \rho>0$ such that:

$$
|g(x, \sigma, \xi)| \geq \rho\left(\sum_{i=1}^{N}\left|\xi_{i}\right|^{p_{i}(x)}\right), \quad \forall \sigma \text { such that }|\sigma|>\rho .
$$

In [1], the authors obtain the existence of renormalized and entropy solutions for the nonlinear elliptic equation with degenerate coercivity of the type

$$
-\operatorname{div}\left[a(x, u)|\nabla u|^{p(x)-2} \nabla u\right]+g(x, u)=f \in L^{1}(\Omega) .
$$

For $g \equiv 0$ and $f \in L^{m(\cdot)}(\Omega)$, with $m(x) \geq m_{-} \geq 1$, equation of the from (1.1) have been widely studied in [2], where the authors obtain some existence and regularity results for the solutions. If $g \equiv|u|^{s(x)-1} u$,

$$
a_{i}(x, u, \nabla u)=\frac{\left|D_{i} u\right|^{p_{i}(x)-2} D_{i} u}{(1+|u|)^{\gamma_{i}(x)}}
$$


and $f \in L^{m}(\Omega)$, with $m \geq 1$, existence and regularity results of distributional solutions have been proved in [3].

As far as the existence results for our problem (1.1) there are three difficulties associated with this kind of problems. Firstly, from hypothesis (1.2), the operator

$$
A u=-\sum_{i=1}^{N} D_{i} a_{i}(x, u, \nabla u)
$$

is well defined between $W_{0}^{1, \vec{p}(\cdot)}(\Omega)$ and its dual space $\left(W_{0}^{1, \vec{p}(\cdot)}(\Omega)\right)^{\prime}$. However, by assumption (1.4), if we take for example

$$
\left\{\begin{array}{l}
a_{i}\left(x, u_{n}, \nabla u_{n}\right)=\frac{\left|D_{i} u_{n}\right|^{p_{i}(x)-2} D_{i} u_{n}}{\left(1+\left|u_{n}\right|\right)^{\gamma_{i}(x)}} \\
u_{n}(x)=|x|^{\frac{n\left(p_{+}^{+}-N\right)}{(n+1) p_{+}^{+}}}-1, \quad|x| \leq 1
\end{array} \quad \text { where } p_{+}^{+}\right. \text {is defined as in (2.3), }
$$

the operator $A$ is not coercive. Because, if $\left\|u_{n}\right\|_{W^{1, \vec{p}(\cdot)}(\Omega)}$ tends to infinity then

$$
\frac{\left\langle A u_{n}, u_{n}\right\rangle}{\left\|u_{n}\right\|_{W^{1, \vec{p}(\cdot)}(\Omega)}} \rightarrow 0 .
$$

So, the classical methods used in order to prove the existence of a solution for (1.1) cannot be applied. The second difficulty is represented in the fact that $g(x, u, \nabla u)$ can not be defined from $W^{1, \vec{p}(\cdot)}(\Omega)$ into its dual, but from $W^{1, \vec{p}(\cdot)}(\Omega) \cap L^{\infty}(\Omega)$ into $L^{1}(\Omega)$. The third difficulty appears when we give a variable exponential growth condition (1.2) for $a_{i}$. The operator $A$ possesses more complicated nonlinearities; thus, some techniques used in the constant exponent case cannot be carried out for the variable exponent case. For more recent results for elliptic and parabolic case, see the papers [4-8] and references therein.

The paper is organized as follows. In Section 2, we present results on the Lebesgue and Sobolev spaces with variable exponents both for the isotropic and the anisotropic cases, and state the main results. The proof of the main result will be presented in Section 3 . We start by giving an existence result for an approximate problem associated with (1.1). The second part of Section 3 is devoted to proving the main existence result by using a priori estimates and then passing to the limit in the approximate problem.

\section{Preliminaries and statement of the main result}

\subsection{Preliminaries}

In this sub-section, we recall some facts on anisotropic spaces with variable exponents and we give some of their properties. For further details on the Lebesgue-Sobolev spaces 
with variable exponents, we refer to [9-11] and references therein. Let $\Omega$ be a bounded open subset of $\mathbb{R}^{N}(N \geq 2)$, we denote

$$
p^{+}=\max _{x \in \bar{\Omega}} p(x), \quad p^{-}=\min _{x \in \bar{\Omega}} p(x)
$$

and

$$
C_{+}(\bar{\Omega})=\left\{p \in C(\bar{\Omega}) \mid p^{-}>1\right\} .
$$

Let $p(\cdot) \in C_{+}(\bar{\Omega})$. We define the space

$$
L^{p(\cdot)}(\Omega)=\left\{u: \Omega \rightarrow \mathbb{R}^{N} \text { measurable }\left.\left|\int_{\Omega}\right| u(x)\right|^{p(x)} \mathrm{d} x<\infty\right\}
$$

then the expression

$$
\|u\|_{p(\cdot)}:=\|u\|_{L^{p(\cdot)}(\Omega)}=\inf \left\{\lambda>\left.0\left|\int_{\Omega}\right| \frac{u(x)}{\lambda}\right|^{p(x)} \mathrm{d} x \leq 1\right\},
$$

defines a norm in $L^{p(\cdot)}(\Omega)$, called the Luxemburg norm. The space $\left(L^{p(\cdot)}(\Omega),\|u\|_{p(\cdot)}\right)$ is a separable Banach space. If $0<\operatorname{meas}(\Omega)<+\infty$ and $p_{1}, p_{2} \in C_{+}(\bar{\Omega})$ with $p_{1} \leq p_{2}$ in $\Omega$, then the embedding $L^{p_{2}(\cdot)}(\Omega) \hookrightarrow L^{p_{1}(\cdot)}(\Omega)$ is continuous. Moreover, if $1<p^{-}<p^{+}<+\infty$, then $L^{p(\cdot)}(\Omega)$ is uniformly convex, hence reflexive and its dual space is isomorphic to $L^{p^{\prime}(\cdot)}(\Omega)$ where $\frac{1}{p(\cdot)}+\frac{1}{p^{\prime}(\cdot)}=1$. For all $u \in L^{p(\cdot)}(\Omega)$ and $v \in L^{p^{\prime}(\cdot)}(\Omega)$, the Hölder type inequality

$$
\left|\int_{\Omega} u v \mathrm{~d} x\right| \leq\left(\frac{1}{p^{-}}+\frac{1}{p^{\prime-}}\right)\|u\|_{p(\cdot)}\|v\|_{p^{\prime}(\cdot)} \leq 2\|u\|_{p(\cdot)}\|v\|_{p^{\prime}(\cdot)},
$$

holds true. We define the variable exponents Sobolev spaces by

$$
W^{1, p(\cdot)}(\Omega)=\left\{u \in L^{p(\cdot)}(\Omega) \quad|| \nabla u \mid \in L^{p(\cdot)}(\Omega)\right\},
$$

which is a Banach space equipped with the following norm

$$
\|u\|_{1, p(\cdot)}=\|u\|_{p(\cdot)}+\|\nabla u\|_{p(\cdot) \cdot} .
$$

Next, we define $W_{0}^{1, p(\cdot)}(\Omega)$ as the closure of $C_{0}^{\infty}(\Omega)$ in $W^{1, p(\cdot)}(\Omega)$. Finally, we introduce a natural generalization of the variable exponents Sobolev spaces $W_{0}^{1, p(\cdot)}(\Omega)$ that will enable us to study with sufficient accuracy problem (1.1). Let $\vec{p}(\cdot)=\left(p_{1}(\cdot), \ldots, p_{N}(\cdot)\right)$, where $p_{i}: \bar{\Omega} \rightarrow(1,+\infty)$ are continuous functions. We introduce the anisotropic variable exponents Sobolev spaces

$$
W^{1, \vec{p}(\cdot)}(\Omega)=\left\{u \in L^{p_{i}(\cdot)}(\Omega) \mid D_{i} u \in L^{p_{i}(\cdot)}(\Omega), i=1, \ldots, N\right\},
$$


with respect to the norm

$$
\|v\|_{1, \vec{p}(\cdot)}=\sum_{i=1}^{N}\left(\|u\|_{L^{p_{i}(\cdot)}(\Omega)}+\left\|D_{i} u\right\|_{L^{p_{i}(\cdot)}(\Omega)}\right)
$$

We introduce the following notation $p_{+}^{+}, p_{-}^{-} \in \mathbb{R}^{+}$as

$$
p_{+}^{+}=\max \left\{p_{1}^{+}, \ldots, p_{N}^{+}\right\}, \quad p_{-}^{-}=\min \left\{p_{1}^{-}, \ldots, p_{N}^{-}\right\} .
$$

We denote $W_{0}^{1, \vec{p}(\cdot)}(\Omega)=W_{0}^{1,1}(\Omega) \cap W^{1, \vec{p}(\cdot)}(\Omega)$ with respect to the norm (2.2). According to [10], $W_{0}^{1, \vec{p}(\cdot)}(\Omega)$ is a reflexive Banach space.

Theorem 2.1 ([10]). Let $\Omega \subset \mathbb{R}^{N}$ be a bounded domain and $\vec{p}(\cdot)=\left(p_{1}(\cdot), p_{2}(\cdot), \ldots, p_{N}(\cdot)\right) \in$ $\left(C_{+}(\bar{\Omega})\right)^{N}$. Suppose that

$$
p^{+}(x)<\bar{p}^{*}(x) \text { for all } x \in \bar{\Omega} \text {. }
$$

Then

$$
\|u\|_{L^{p^{+}(\cdot)}(\Omega)} \leq C \sum_{i=1}^{N}\left\|D_{i} u\right\|_{L^{p_{i}(\cdot)}(\Omega)^{\prime}} \quad \forall u \in W_{0}^{1, \vec{p}(\cdot)}(\Omega),
$$

where $p^{+}$is defined as in (2.1), $\bar{p}^{*}$ as in (1.5), and $C$ is a positive constant independent of $u$. Thus $\sum_{i=1}^{N}\left\|D_{i} u\right\|_{L^{p_{i}(\cdot)}(\Omega)}$ is an equivalent norm on $W_{0}^{1, \vec{p}(\cdot)}(\Omega)$.

Proposition 2.1. Suppose that the hypotheses of Theorem 2.1 are satisfied. Then, for all $u \in$ $W_{0}^{1, \vec{p}(\cdot)}(\Omega)$ we have

$$
\frac{1}{N^{p_{-}^{-}-1}}\|u\|_{1, \vec{p}(\cdot)}^{p_{-}^{-}}-N \leq \sum_{i=1}^{N} \int_{\Omega}\left|D_{i} u\right|^{p_{i}(x)} \mathrm{d} x \leq N+\|u\|_{1, \vec{p}(\cdot)}^{p_{+}^{+}} .
$$

Proof. Put

$$
\mathcal{I}=\left\{i \in\{1, \ldots, N\} \mid\left\|D_{i} u\right\|_{p_{i}(\cdot)} \leq 1\right\} \quad \text { and } \quad \mathcal{J}=\left\{i \in\{1, \ldots, N\} \mid\left\|D_{i} u\right\|_{p_{i}(\cdot)}>1\right\} .
$$

Thanks to (Proposition 2.1 in [3]), we have

$$
\begin{aligned}
\sum_{i=1}^{N} \int_{\Omega}\left|D_{i} u\right|^{p_{i}(x)} \mathrm{d} x & =\sum_{i \in \mathcal{I}} \int_{\Omega}\left|D_{i} u\right|^{p_{i}(x)} \mathrm{d} x+\sum_{i \in \mathcal{J}} \int_{\Omega}\left|D_{i} u\right|^{p_{i}(x)} \mathrm{d} x \\
& \geq \sum_{i \in \mathcal{I}}\left\|D_{i} u\right\|_{p_{i}(\cdot)}^{p_{i}^{+}}+\sum_{i \in \mathcal{J}}\left\|D_{i} u\right\|_{p_{i}(\cdot)}^{p_{i}^{-}} \\
& \geq \sum_{i=1}^{N}\left\|D_{i} u\right\|_{p_{i}(\cdot)}^{p_{-}^{-}}-\sum_{i \in \mathcal{I}}\left\|D_{i} u\right\|_{p_{i}(\cdot)}^{p_{-}^{-}} \geq \sum_{i=1}^{N}\left\|D_{i} u\right\|_{p_{i}(\cdot)}^{p_{-}^{-}}-N .
\end{aligned}
$$


Using the convexity of the application $t \in \mathbb{R}^{+} \mapsto t^{p_{-}^{-}}, p_{-}^{-}>1$, we obtain

$$
\begin{aligned}
& \sum_{i=1}^{N} \int_{\Omega}\left|D_{i} u\right|^{p_{i}(x)} \mathrm{d} x \geq \frac{1}{N^{p_{-}^{-}-1}}\|u\|_{1, \bar{p}(\cdot)}^{p_{-}^{-}}-N, \\
& \sum_{i=1}^{N} \int_{\Omega}\left|D_{i} u\right|^{p_{i}(x)} \mathrm{d} x \leq \sum_{i \in \mathcal{I}}\left\|D_{i} u\right\|_{p_{i}(\cdot)}^{p_{i}^{-}}+\sum_{i \in \mathcal{J}}\left\|D_{i} u\right\|_{p_{i}(\cdot)}^{p_{i}^{+}} \leq N+\sum_{i=1}^{N}\left\|D_{i} u\right\|_{p_{i}(\cdot)}^{p_{+}^{+}} .
\end{aligned}
$$

We will use through the paper, the truncation function $T_{k}$ at height $k(k>0)$, that is $T_{k}(s):=\max \{-k, \min \{k, s\}\}$.

Lemma 2.1 ([12]). Let $g \in L^{p(\cdot)}(\Omega)$ and $g_{n} \in L^{p(\cdot)}(\Omega)$ with $\left\|g_{n}\right\|_{p(\cdot)} \leq$ C. If $g_{n}(x) \rightarrow g(x)$ almost everywhere in $\Omega$, then $g_{n} \rightarrow g$ in $L^{p(\cdot)}(\Omega)$.

Lemma $2.2([13])$. Let $u \in W_{0}^{1, \vec{p}(\cdot)}(\Omega)$, then $T_{k}(u) \in W_{0}^{1, \vec{p}(\cdot)}(\Omega)$ for all $k>0$. Moreover, we have $T_{k}(u) \rightarrow u$ in $W_{0}^{1, \vec{p}(\cdot)}(\Omega)$ as $k \rightarrow \infty$.

Lemma 2.3 ([13]). Let $\left(u_{n}\right)_{n}$ be a bounded sequence in $W_{0}^{1, \vec{p}(\cdot)}(\Omega)$. If $u_{n} \rightarrow u$ in $W_{0}^{1, \vec{p}(\cdot)}(\Omega)$, then $T_{k}\left(u_{n}\right) \rightarrow T_{k}(u)$ in $W_{0}^{1, \vec{p}(\cdot)}(\Omega)$.

Lemma 2.4 ([13]). Assume that (1.2)-(1.4) hold and let $\left(u_{n}\right)_{n}$ be a sequence in $W_{0}^{1, \vec{p}(\cdot)}(\Omega)$ such that $u_{n} \rightarrow u$ in $W_{0}^{1, \vec{p}(\cdot)}(\Omega)$ and

$$
\sum_{i=1}^{N} \int_{\Omega}\left(a_{i}\left(x, u_{n}, \nabla u_{n}\right)-a_{i}\left(x, u_{n}, \nabla u\right)\right)\left(D_{i} u_{n}-D_{i} u\right) \mathrm{d} x \rightarrow 0 .
$$

Then, $u_{n} \rightarrow u$ in $W_{0}^{1, \vec{p}(\cdot)}(\Omega)$ for a subsequence.

\subsection{Statement of main result}

We will extend the notion of distributional solution, see $[12,13]$, to problem $(1.1)$ as follows:

Definition 2.1. Let $f \in L^{1}(\Omega)$ a measurable function $u$ is said to be solution in the sense of distributions to the problem (1.1), if

$$
\begin{aligned}
& u \in W_{0}^{1, \vec{p}(\cdot)}(\Omega), g(x, u, \nabla u) \in L^{1}(\Omega), \quad \text { and } \forall v \in W_{0}^{1, \vec{p}(\cdot)}(\Omega) \cap L^{\infty}(\Omega) \\
& \sum_{i=1}^{N} \int_{\Omega} a_{i}(x, u, \nabla u) D_{i} v \mathrm{~d} x+\int_{\Omega} g(x, u, \nabla u) v \mathrm{~d} x=\int_{\Omega} f v \mathrm{~d} x .
\end{aligned}
$$

Our main result is as follows

Theorem 2.2. Let $f \in L^{1}(\Omega)$. Assume (1.2)-(1.8) and (2.4). Then problem (1.1) has at least one solution in the sense of distributions. 


\section{Proof of the main result}

\subsection{Approximate solution}

Let $\left(f_{n}\right)_{n}$ be a sequence in $L^{\infty}(\Omega)$ such that $f_{n} \rightarrow f$ in $L^{1}(\Omega)$ with $\left|f_{n}\right| \leq|f|$ (for example $\left.f_{n}=T_{n}(f)\right)$ and we consider the approximate problem

$$
\left\{\begin{array}{l}
-\sum_{i=1}^{N} D_{i} a_{i}\left(x, T_{n}\left(u_{n}\right), \nabla u_{n}\right)+g\left(x, u_{n}, \nabla u_{n}\right)=T_{n}(f) \text { in } \Omega, \\
u_{n} \in W_{0}^{1, \vec{p}(\cdot)}(\Omega) .
\end{array}\right.
$$

Lemma 3.1. Let $f \in L^{1}(\Omega)$. Assume (1.2)-(1.8) and (2.4). Then, problem (3.1) has at least one solution in the sense of distributions.

Proof. Let us define the operator $A_{n}$ from $W_{0}^{1, \vec{p}(\cdot)}(\Omega)$ into its dual $\left(W_{0}^{1, \vec{p}(\cdot)}(\Omega)\right)^{\prime}$, by

$$
A_{n} u=-\sum_{i=1}^{N} D_{i} a_{i}\left(x, T_{n}(u), \nabla u\right) \quad \text { and } \quad g^{k}(x, s, \xi)=\frac{g(x, s, \xi)}{1+|g(x, s, \xi)| / k} .
$$

Note that $g^{k}(x, s, \xi) s \geq 0,\left|g^{k}(x, s, \xi)\right| \leq|g(x, s, \xi)|$ and $\left|g^{k}(x, s, \xi)\right| \leq k$ for all $k \in \mathbb{N}-\{0\}$. We define $G_{k}: W_{0}^{1, \vec{p}(\cdot)}(\Omega) \rightarrow\left(W_{0}^{1, \vec{p}(\cdot)}(\Omega)\right)^{\prime}$, by

$$
\left\langle G_{k} u, v\right\rangle=\int_{\Omega} g^{k}(x, u, \nabla u) v \mathrm{~d} x .
$$

Consider the following problem

$$
A_{n} u_{n_{k}}+g^{k}\left(x, u_{n_{k}}, \nabla u_{n_{k}}\right)=T_{n}(f) \text { in } \Omega, \quad u_{n_{k}} \in W_{0}^{1, \vec{p}(\cdot)}(\Omega) .
$$

Lemma 3.2. Let $f \in L^{1}(\Omega)$. Assume that (1.2)-(1.8) and (2.4) hold, then the problem (3.2) has at least one solution $u_{n_{k}}$ in the sense of distributions.

Lemma 3.3. The operator $B_{k}^{n}=A_{n}+G_{k}$ from $W_{0}^{1, \vec{p}(\cdot)}(\Omega)$ into $\left(W_{0}^{1, \vec{p}(\cdot)}(\Omega)\right)^{\prime}$ is pseudo-monotone, moreover, $B_{k}^{n}$ is coercive in the following sense

$$
\frac{\left\langle B_{k}^{n} v, v\right\rangle}{\|v\|_{1, \vec{p}(\cdot)}} \rightarrow+\infty \quad \text { if }\|v\|_{1, \vec{p}(\cdot)} \rightarrow+\infty \quad \text { for } v \in W_{0}^{1, \vec{p}(\cdot)}(\Omega)
$$

Proof of the Lemma 3.3. Thanks to the Hölder inequality, we have for all $u, v \in W_{0}^{1, \vec{p}(\cdot)}(\Omega)$

$$
\left|\left\langle G_{k} u, v\right\rangle\right| \leq\left(\frac{1}{p_{i}^{-}}+\frac{1}{\left(p_{i}^{-}\right)^{\prime}}\right)\left\|g^{k}(x, u, \nabla u)\right\|_{p_{i}^{\prime}(\cdot)}\|v\|_{p_{i}(\cdot)}
$$




$$
\begin{aligned}
& \leq C_{0}\left(\frac{1}{\left(p_{-}^{-}\right)}+\frac{1}{\left(p_{-}^{-}\right)^{\prime}}\right)\left(k^{\left(p_{+}^{+}\right)^{\prime}} \operatorname{meas}(\Omega)+1\right)^{\frac{1}{\left(p_{-}^{-}\right)^{\prime}}}\|v\|_{1, \vec{p}(\cdot)} \\
& \leq C_{1}\|v\|_{1, \vec{p}(\cdot)}
\end{aligned}
$$

Thanks to the Hölder inequality and (1.2), we have for all $u, v \in W_{0}^{1, \vec{p}(\cdot)}(\Omega)$

$$
\begin{aligned}
\left|\left\langle A_{n} u, v\right\rangle\right| & \leq 2 \sum_{i=1}^{N}\left\|\left(k(x)+\left|T_{n}(u)\right|^{\bar{p}(x)}+\sum_{j=1}^{N}\left|D_{i} u\right|^{p_{j}(x)}\right)^{1-\frac{1}{p_{i}(x)}}\right\|_{p_{i}^{\prime}(\cdot)}\left\|D_{i} v\right\|_{p_{i}(\cdot)} \\
& \leq C_{2}\|v\|_{1, \vec{p}(\cdot)}
\end{aligned}
$$

Then by using (3.3) and (3.4) we conclude that $B_{k}^{n}=A_{n}+G_{k}$ is bounded. For the coercivity, by using (1.4), (1.7), and (2.5), we get

$$
\begin{aligned}
\left\langle B_{k}^{n} u, u\right\rangle & \geq \sum_{i=1}^{N} \int_{\Omega} a_{i}\left(x, T_{n}(u), \nabla u\right) D_{i} u \mathrm{~d} x \geq \sum_{i=1}^{N} \int_{\Omega} \frac{\alpha}{(1+n)^{\gamma_{i}(x)}}\left|D_{i} u\right|^{p_{i}(x)} \mathrm{d} x \\
& \geq \frac{C_{3}}{N^{p_{-}^{-}-1}}\|u\|_{1, \vec{p}(\cdot)}^{p_{-}^{-}}-C_{3} N,
\end{aligned}
$$

then

$$
\frac{\left\langle B_{k}^{n} u, u\right\rangle}{\|u\|_{1, \vec{p}(\cdot)}} \geq \frac{C_{3}}{N^{p_{-}^{-}-1}}\|u\|_{1, \vec{p}(\cdot)}^{p_{-}^{-}-1}-\frac{C_{3} N}{\|u\|_{1, \vec{p}(\cdot)}} \rightarrow+\infty \quad \text { as }\|u\|_{1, \vec{p}(\cdot)} \rightarrow+\infty .
$$

It remains to show that $B_{k}^{n}$ is pseudo-monotone. Let $\left(u_{m}\right)_{m}$ be a sequence in $W_{0}^{1, \vec{p}(\cdot)}(\Omega)$ such that

$$
\begin{cases}u_{m} \rightarrow u, & \text { in } W_{0}^{1, \vec{p}(\cdot)}(\Omega), \\ B_{k}^{n} u_{m} \rightarrow \chi_{k}^{n}, & \text { in }\left(W_{0}^{1, \vec{p}(\cdot)}(\Omega)\right)^{\prime}, \\ \limsup _{m \rightarrow \infty}\left\langle B_{k}^{n} u_{m}, u_{m}\right\rangle \leq\left\langle\chi_{k}^{n}, u\right\rangle .\end{cases}
$$

We will prove that

$$
\chi_{k}^{n}=B_{k}^{n} u \quad \text { and } \quad\left\langle B_{k}^{n} u_{m}, u_{m}\right\rangle \rightarrow\left\langle\chi_{k}^{n}, u\right\rangle \quad \text { as } m \rightarrow+\infty .
$$

Firstly, since $W_{0}^{1, \vec{p}(\cdot)}(\Omega)$ is compactly embedded in $L^{p_{-}^{-}}(\Omega)$, then $u_{m} \rightarrow u$ in $L^{p_{-}^{-}}(\Omega)$ and

$$
u_{m} \rightarrow u \text { a.e. in } \Omega,
$$

for a subsequence still denoted $\left(u_{m}\right)_{m}$. The sequence $\left(u_{m}\right)_{m}$ is bounded in $W_{0}^{1, \vec{p}(\cdot)}(\Omega)$. Then, by (1.2) we have $a_{i}\left(x, T_{n}\left(u_{m}\right), \nabla u_{m}\right)$ is bounded in $L^{p_{i}^{\prime}(\cdot)}(\Omega)$. Therefore, there exists a function $\varphi_{i}^{n} \in L^{p_{i}^{\prime}(\cdot)}(\Omega)$ such that

$$
a_{i}\left(x, T_{n}\left(u_{m}\right), \nabla u_{m}\right) \rightarrow \varphi_{i}^{n} \quad \text { in } \quad L^{p_{i}^{\prime}(\cdot)}(\Omega) \quad \text { as } m \rightarrow \infty .
$$


Similarly, since $\left(g^{k}\left(x, u_{m}, \nabla u_{m}\right)\right)_{m}$ is bounded in $L^{\left(p_{-}^{-}\right)^{\prime}}(\Omega)$ with $\left(p_{-}^{-}\right)^{\prime}$ is the conjugate exponent of $\left(p_{-}^{-}\right)$, there exists a function $\psi^{k} \in L^{\left(p_{-}^{-}\right)^{\prime}}(\Omega)$ such that

$$
g^{k}\left(x, u_{m}, \nabla u_{m}\right) \rightarrow \psi^{k} \quad \text { in } \quad L^{\left(p_{-}^{-}\right)^{\prime}}(\Omega) \quad \text { as } m \rightarrow \infty .
$$

For all $v \in W_{0}^{1, \vec{p}(\cdot)}(\Omega)$, we have

$$
\left\langle\chi_{k}^{n}, v\right\rangle=\sum_{i=1}^{N} \int_{\Omega} \varphi_{i}^{n} D_{i} v \mathrm{~d} x+\int_{\Omega} \psi^{k} v \mathrm{~d} x .
$$

Using (3.5), (3.8), (3.9), and that $u_{m} \rightarrow u$ in $L^{p_{-}^{-}}(\Omega)$, we have

$$
\int_{\Omega} g^{k}\left(x, u_{m}, \nabla u_{m}\right) u_{m} \mathrm{~d} x \rightarrow \int_{\Omega} \psi^{k} u \mathrm{~d} x,
$$

therefore, thanks to (3.5), (3.9), and (3.10), we write

$$
\underset{m \rightarrow \infty}{\limsup } \sum_{i=1}^{N} \int_{\Omega} a_{i}\left(x, T_{n}\left(u_{m}\right), \nabla u_{m}\right) D_{i} u_{m} \mathrm{~d} x \leq \sum_{i=1}^{N} \int_{\Omega} \varphi_{i}^{n} D_{i} u \mathrm{~d} x .
$$

On the other hand, by (1.3), we obtain

$$
\begin{aligned}
& \quad \sum_{i=1}^{N} \int_{\Omega} a_{i}\left(x, T_{n}\left(u_{m}\right), \nabla u_{m}\right) D_{i} u_{m} \mathrm{~d} x \\
& \geq \sum_{i=1}^{N} \int_{\Omega} a_{i}\left(x, T_{n}\left(u_{m}\right), \nabla u_{m}\right) D_{i} u \mathrm{~d} x \\
& \quad+\sum_{i=1}^{N} \int_{\Omega} a_{i}\left(x, T_{n}\left(u_{m}\right), \nabla u\right)\left(D_{i} u_{m}-D_{i} u\right) \mathrm{d} x .
\end{aligned}
$$

In view of Lebesgue dominated convergence theorem and (3.6), we have

$$
a_{i}\left(x, T_{n}\left(u_{m}\right), \nabla u\right) \rightarrow a_{i}\left(x, T_{n}(u), \nabla u\right) \quad \text { in } L^{p_{i}^{\prime}(\cdot)}(\Omega) .
$$

By (3.7) and (3.5), we get

$$
\liminf _{m \rightarrow \infty} \sum_{i=1}^{N} \int_{\Omega} a_{i}\left(x, T_{n}\left(u_{m}\right), \nabla u_{m}\right) D_{i} u_{m} \mathrm{~d} x \geq \sum_{i=1}^{N} \int_{\Omega} \varphi_{i}^{n} D_{i} u \mathrm{~d} x,
$$

this implies, thanks to (3.11), that

$$
\lim _{m \rightarrow \infty} \sum_{i=1}^{N} \int_{\Omega} a_{i}\left(x, T_{n}\left(u_{m}\right), \nabla u_{m}\right) D_{i} u_{m} \mathrm{~d} x=\sum_{i=1}^{N} \int_{\Omega} \varphi_{i}^{n} D_{i} u \mathrm{~d} x .
$$


By combining (3.5), (3.10), and (3.12) we deduce that $\left\langle B_{k}^{n} u_{m}, u_{m}\right\rangle \rightarrow\left\langle\chi_{k}^{n}, u\right\rangle$ as $m \rightarrow \infty$. Now, by (3.12) we obtain

$$
\lim _{m \rightarrow \infty} \sum_{i=1}^{N} \int_{\Omega}\left(a_{i}\left(x, T_{n}\left(u_{m}\right), \nabla u_{m}\right)-a_{i}\left(x, T_{n}\left(u_{m}\right), \nabla u\right)\right)\left(D_{i} u_{m}-D_{i} u\right) \mathrm{d} x=0 .
$$

In view of Lemma 2.4, we get $u_{m} \rightarrow u$ in $W_{0}^{1, \vec{p}(\cdot)}(\Omega)$ and $D_{i} u_{m} \rightarrow D_{i} u$ almost everywhere in $\Omega$, then $a_{i}\left(x, T_{n}\left(u_{m}\right), \nabla u_{m}\right) \rightarrow a_{i}\left(x, T_{n}(u), \nabla u\right)$ in $L^{p_{i}^{\prime}(\cdot)}(\Omega)$ and $g^{k}\left(x, u_{m}, \nabla u_{m}\right) \rightarrow g^{k}(x, u, \nabla u)$ in $L^{\theta^{\prime}(\cdot)}(\Omega)$ for all $i=1, \ldots, N$, where $\theta^{\prime}(x) \geq \max \left\{p_{i}(\cdot), \quad i=1, \ldots, N\right\}$, so we deduce that $\chi_{k}^{n}=B_{k}^{n} u$, which completes the proof of Lemma 3.3.

Proof of the Lemma 3.2. In view of Lemma 3.3, there exists at least one weak solution $u_{n_{k}} \in$ $W_{0}^{1, \vec{p}(\cdot)}(\Omega)$ of problem (3.2) (see [14]).

Lemma 3.4. Let $f \in L^{1}(\Omega)$, assume that (1.2)-(1.7) and (2.4) hold. Let $u_{n_{k}} \in W_{0}^{1, \vec{p}(\cdot)}(\Omega)$ be a distribution solution of (3.2). Then, there exists a constant $C(n)>0$ such that

$$
\sum_{i=1}^{N} \int_{\Omega}\left|D_{i} u_{n_{k}}\right|^{p_{i}(x)} \mathrm{d} x \leq C(n) .
$$

Proof. The proof uses the same technique as in (Lemma 4.1 of [3]) and is omited here.

Therefore, by Lemma 3.4 the sequence $\left\{u_{n_{k}}\right\}_{k}$ is bounded in $W_{0}^{1, \vec{p}(\cdot)}(\Omega)$. As a consequence, there exists a function $u_{n} \in W_{0}^{1, \vec{p}(\cdot)}(\Omega)$ and a subsequence (still denoted by $u_{n_{k}}$ ) such that

$$
u_{n_{k}} \rightarrow u_{n} \quad \text { weakly in } \quad W_{0}^{1, \vec{p}(\cdot)}(\Omega) \text { and a.e. in } \Omega \text { as } k \rightarrow \infty .
$$

Lemma 3.5. Assume that hypotheses (1.2), (1.7), and (2.4) hold. Let $u_{n_{k}} \in W_{0}^{1, \vec{p}(\cdot)}(\Omega)$ be a distribution solution of (3.2). Then, there exist a subsequence of $\left(u_{n_{k}}\right)$ denoted by itself, and a measurable function $u_{n} \in W_{0}^{1, \vec{p}(\cdot)}(\Omega)$, such that

$$
T_{h}\left(u_{n_{k}}\right) \rightarrow T_{h}\left(u_{n}\right) \quad \text { in } W_{0}^{1, \vec{p}(\cdot)}(\Omega) .
$$

Proof. It is similar to the proof of Theorem 4.2 of [13].

\subsection{A priori estimates}

Lemma 3.6. Assume (1.2)-(2.4). Let $u_{n} \in W_{0}^{1, \vec{p}(\cdot)}(\Omega)$ be a distribution solution of (1.1). Then, there exists a constant $C \geq 0$ such that

$$
\left\|u_{n}\right\|_{1, \vec{p}(\cdot)} \leq C
$$


Proof. Let $h>0$. Taking $T_{h}\left(u_{n}\right)$ as a test function in (3.1), then

$$
\begin{aligned}
& \sum_{i=1}^{N} \int_{\Omega} a_{i}\left(x, T_{n}\left(u_{n}\right), \nabla u_{n}\right) D_{i} T_{h}\left(u_{n}\right) \mathrm{d} x+\int_{\Omega} g\left(x, u_{n}, \nabla u_{n}\right) T_{h}\left(u_{n}\right) \mathrm{d} x \\
= & \int_{\Omega} T_{n}(f) T_{h}\left(u_{n}\right) \mathrm{d} x .
\end{aligned}
$$

By dropping the nonnegative term in (3.13), (1.7), and (1.4) we get

$$
\sum_{i=1}^{N} \int_{\Omega} \frac{\alpha}{\left(1+\left|T_{n}\left(u_{n}\right)\right|\right)^{\gamma_{i}(x)}}\left|D_{i} T_{h}\left(u_{n}\right)\right|^{p_{i}(x)} \mathrm{d} x \leq h \int_{\Omega}|f| \mathrm{d} x,
$$

then

$$
\sum_{i=1}^{N} \int_{\Omega} \frac{\alpha}{(1+h)^{\gamma_{i}(x)}}\left|D_{i} T_{h}\left(u_{n}\right)\right|^{p_{i}(x)} \mathrm{d} x \leq h\|f\|_{L^{1}(\Omega)}
$$

Consequently,

$$
\sum_{i=1}^{N} \int_{\Omega}\left|D_{i} T_{h}\left(u_{n}\right)\right|^{p_{i}(x)} \mathrm{d} x \leq C_{3} .
$$

Taking $T_{h}\left(u_{n}\right)$ as a test function in (3.1), and dropping the first nonnegative term in the left-hand side, we obtain

$$
\int_{\left\{\left|u_{n}\right|>h\right\}}\left|g\left(x, u_{n}, \nabla u_{n}\right)\right| \mathrm{d} x \leq\|f\|_{L^{1}(\Omega)} .
$$

By combining (1.8), (3.14) and (3.15), for $h=\rho$, we deduce that

$$
\sum_{i=1}^{N} \int_{\Omega}\left|D_{i} u_{n}\right|^{p_{i}(x)} \mathrm{d} x \leq C_{4}+\frac{1}{h} \int_{\left\{\left|u_{n}\right|>h\right\}}\left|g\left(x, u_{n}, \nabla u_{n}\right)\right| \mathrm{d} x \leq C_{4}+\frac{\|f\|_{L^{1}(\Omega)}}{h}=C_{5} .
$$

By (2.5), we get $\left\|u_{n}\right\|_{1, \vec{p}(\cdot)} \leq C_{6}$. Consequently, there exist a subsequence of $u_{n}$ (denoted by itself) and a measurable function $u \in W_{0}^{1, \vec{p}(\cdot)}(\Omega)$, such that

$$
\begin{cases}u_{n} \rightarrow u, & \text { in } W_{0}^{1, \vec{p}(\cdot)}(\Omega), \\ u_{n} \rightarrow u, & \text { in } L^{p_{-}^{-}}(\Omega)\end{cases}
$$

This ends the proof of Lemma 3.6. 


\subsection{The strong convergence of the truncation}

Lemma 3.7. Assume that hypotheses (1.2)-(1.8) and (2.4) hold, and let $u_{n} \in W_{0}^{1, \vec{p}(\cdot)}(\Omega)$ be a distribution solution of (1.1). Then, there exist a subsequence of $u_{n}$ denoted by itself, and $a$ measurable function $u \in W_{0}^{1, \vec{p}(\cdot)}(\Omega)$ such that

$$
T_{j}\left(u_{n}\right) \rightarrow T_{j}(u) \quad \text { strongly in } W_{0}^{1, \vec{p}(\cdot)}(\Omega) .
$$

Proof. Let $h \geq j>0$ and $w_{n}=T_{2 j}\left(u_{n}-T_{h}\left(u_{n}\right)+T_{j}\left(u_{n}\right)-T_{j}(u)\right)$. We set $\varphi_{j}(s)=s \cdot \exp \left(\delta s^{2}\right)$, where $\delta=(l(j) /(2 \alpha))^{2}, l(j)=b(j)(1+|j|)^{\gamma_{+}^{+}}$, and

$$
\varphi_{j}^{\prime}(s)-\frac{l(j)}{\alpha}\left|\varphi_{j}(s)\right| \geq \frac{1}{2}, \quad \forall s \in \mathbb{R} .
$$

Let $M=4 j+h$. Since $D_{i} w_{n}=0$ on $\left\{\left|u_{n}\right|>M\right\}$ and $\varphi_{j}\left(w_{n}\right)$ has the same sign as $u_{n}$ on the set $\left\{\left|u_{n}\right|>j\right\}$ ( indeed, if $u_{n}>j$ then $u_{n}-T_{h}\left(u_{n}\right) \geq 0$ and $T_{j}\left(u_{n}\right)-T_{j}(u) \geq 0$, it follows that $\left.w_{n} \geq 0\right)$. Similarly, we show that $w_{n} \leq 0$ on the set $\left\{u_{n}<-j\right\}$.

By taking $\varphi_{j}\left(w_{n}\right)$ as a test function in (3.1), we obtain

$$
\begin{aligned}
& \sum_{i=1}^{N} \int_{\Omega} a_{i}\left(x, T_{M}\left(u_{n}\right), \nabla T_{M}\left(u_{n}\right)\right) \varphi_{j}^{\prime}\left(w_{n}\right) D_{i} w_{n} \mathrm{~d} x \\
& \quad+\int_{\left|u_{n}\right| \leq j} g\left(x, u_{n}, \nabla u_{n}\right) \varphi_{j}\left(w_{n}\right) \mathrm{d} x \leq \int_{\Omega} T_{n}(f) \varphi_{j}\left(w_{n}\right) \mathrm{d} x
\end{aligned}
$$

Taking $y_{n}=u_{n}-T_{h}\left(u_{n}\right)+T_{k}\left(u_{n}\right)-T_{k}(u)$, we have

$$
\begin{aligned}
& \sum_{i=1}^{N} \int_{\Omega} a_{i}\left(x, T_{M}\left(u_{n}\right), \nabla T_{M}\left(u_{n}\right)\right) \varphi_{k}^{\prime}\left(w_{n}\right) D_{i} w_{n} \mathrm{~d} x \\
& \geq \sum_{i=1}^{N} \int_{\Omega} a_{i}\left(x, T_{k}\left(u_{n}\right), \nabla T_{k}\left(u_{n}\right)\right) \varphi_{k}^{\prime}\left(w_{n}\right)\left(D_{i} T_{k}\left(u_{n}\right)-D_{i} T_{k}(u)\right) \mathrm{d} x \\
& \quad+\sum_{i=1_{\left\{\left|u_{n}\right|>k\right\}}}^{N} a_{i}\left(x, T_{k}\left(u_{n}\right), \nabla T_{k}\left(u_{n}\right)\right) \varphi_{k}^{\prime}\left(w_{n}\right) D_{i} T_{k}(u) \mathrm{d} x \\
& \quad-\varphi_{k}^{\prime}(2 k) \sum_{i=1_{\left\{\left|u_{n}\right|>k\right\}}} \int_{l}\left|a_{i}\left(x, T_{M}\left(u_{n}\right), \nabla T_{M}\left(u_{n}\right)\right)\right|\left|D_{i} T_{k}(u)\right| \mathrm{d} x,
\end{aligned}
$$

that is equivalent to

$$
\sum_{i=1}^{N} \int_{\Omega}\left(a_{i}\left(x, T_{j}\left(u_{n}\right), \nabla T_{j}\left(u_{n}\right)\right)-a_{i}\left(x, T_{j}\left(u_{n}\right), \nabla T_{j}(u)\right)\right)
$$




$$
\begin{gathered}
\times\left(D_{i} T_{j}\left(u_{n}\right)-D_{i} T_{j}(u)\right) \varphi_{j}^{\prime}\left(w_{n}\right) \mathrm{d} x \\
\leq \sum_{i=1}^{N} \int_{\Omega} a_{i}\left(x, T_{M}\left(u_{n}\right), \nabla T_{M}\left(u_{n}\right)\right) \varphi_{j}^{\prime}\left(w_{n}\right) D_{i} w_{n} \mathrm{~d} x+(\mathbf{A})+(\mathbf{B})+(\mathbf{C}),
\end{gathered}
$$

where
(A) $-\sum_{i=1}^{N} \int_{\left\{\left|u_{n}\right|>k\right\}} a_{i}\left(x, T_{k}\left(u_{n}\right), \nabla T_{k}\left(u_{n}\right)\right) \varphi_{k}^{\prime}\left(w_{n}\right) D_{i} T_{k}(u) \mathrm{d} x$,
(B) $+\varphi_{j}^{\prime}(2 j) \sum_{i=1}^{N} \int_{\left\{\left|u_{n}\right|>j\right\}}\left|a_{i}\left(x, T_{M}\left(u_{n}\right), \nabla T_{M}\left(u_{n}\right)\right)\right|\left|D_{i} T_{j}(u)\right| \mathrm{d} x$,
(C) $-\sum_{i=1}^{N} \int_{\Omega} a_{i}\left(x, T_{j}\left(u_{n}\right), \nabla T_{j}(u)\right)\left(D_{i} T_{j}\left(u_{n}\right)-D_{i} T_{j}(u)\right) \varphi_{j}^{\prime}\left(w_{n}\right) \mathrm{d} x$.

Arguing as in [13], we can prove that

$$
(\mathbf{A})=\varepsilon_{1}(n), \quad(\mathbf{B})=\varepsilon_{2}(n) \quad \text { and } \quad(\mathbf{C})=\varepsilon_{3}(n) .
$$

By (3.16) and (3.17) we conclude that

$$
\begin{aligned}
& \sum_{i=1}^{N} \int_{\Omega}\left(a_{i}\left(x, T_{j}\left(u_{n}\right), \nabla T_{j}\left(u_{n}\right)\right)-a_{i}\left(x, T_{j}\left(u_{n}\right), \nabla T_{j}(u)\right)\right) \\
& \times\left(D_{i} T_{j}\left(u_{n}\right)-D_{i} T_{j}(u)\right) \varphi_{j}^{\prime}\left(w_{n}\right) \mathrm{d} x \\
\leq & \sum_{i=1}^{N} \int_{\Omega} a_{i}\left(x, T_{M}\left(u_{n}\right), \nabla T_{M}\left(u_{n}\right)\right) \varphi_{j}^{\prime}\left(w_{n}\right) D_{i} w_{n} \mathrm{~d} x+\varepsilon_{4}(n) .
\end{aligned}
$$

Using (3.18) and arguing as in [13], we get

$$
\begin{aligned}
& \frac{l(j)}{\alpha} \sum_{i=1}^{N} \int_{\Omega}\left(a_{i}\left(x, T_{j}\left(u_{n}\right), \nabla T_{j}\left(u_{n}\right)\right)-a_{i}\left(x, T_{j}\left(u_{n}\right), \nabla T_{j}(u)\right)\right) \\
& \times\left(D_{i} T_{j}\left(u_{n}\right)-D_{i} T_{j}(u)\right)\left|\varphi_{j}\left(w_{n}\right)\right| \mathrm{d} x \\
\geq & \left|\int_{\left\{\left|u_{n}\right| \leq j\right\}} g_{i}\left(x, T_{j}\left(u_{n}\right), \nabla T_{j}\left(u_{n}\right)\right) \varphi_{j}\left(w_{n}\right) \mathrm{d} x\right|+\varepsilon_{5}(n) .
\end{aligned}
$$

Thanks to (3.18) and (3.19), we obtain

$$
\begin{aligned}
& \frac{1}{2} \sum_{i=1}^{N} \int_{\Omega}\left(a_{i}\left(x, T_{j}\left(u_{n}\right), \nabla T_{j}\left(u_{n}\right)\right)-a_{i}\left(x, T_{j}\left(u_{n}\right), \nabla T_{j}(u)\right)\right) \times\left(D_{i} T_{j}\left(u_{n}\right)-D_{i} T_{j}(u)\right) \mathrm{d} x \\
\leq & \int_{\Omega} T_{n}(f) \varphi_{j}\left(T_{2 j}\left(u-T_{h}(u)\right)\right) \mathrm{d} x+\varepsilon_{6}(n) .
\end{aligned}
$$


Then by letting $h$ tends to infinity in the previous inequality, we get

$$
\begin{aligned}
& \frac{1}{2} \sum_{i=1}^{N} \int_{\Omega}\left(a_{i}\left(x, T_{j}\left(u_{n}\right), \nabla T_{j}\left(u_{n}\right)\right)-a_{i}\left(x, T_{j}\left(u_{n}\right), \nabla T_{j}(u)\right)\right) \\
& \quad \times\left(D_{i} T_{j}\left(u_{n}\right)-D_{i} T_{j}(u)\right) \mathrm{d} x \rightarrow 0, \quad \text { as } n \rightarrow \infty .
\end{aligned}
$$

Using Lemma 2.4, we deduce that $T_{j}\left(u_{n}\right) \rightarrow T_{j}(u)$ in $W_{0}^{1, \vec{p}(\cdot)}(\Omega)$.

Thanks to Lemma 2.2, we obtain

$$
\begin{cases}u_{n} \rightarrow u, & \text { in } W_{0}^{1, \vec{p}(\cdot)}(\Omega), \\ \nabla u_{n} \rightarrow \nabla u, & \text { a.e in } \Omega\end{cases}
$$

\subsection{The equi-integrability of $g\left(x, u_{n}, \nabla u_{n}\right)$ and passage to the limit}

Thanks to (3.20), we have

$$
\begin{array}{ll}
a_{i}\left(x, T_{n}\left(u_{n}\right), \nabla u_{n}\right) \rightarrow a_{i}(x, u, \nabla u) & \text { a.e. in } \Omega, \\
g\left(x, u_{n}, \nabla u_{n}\right) \rightarrow g(x, u, \nabla u) & \text { a.e. in } \Omega .
\end{array}
$$

Using that $\left(a_{i}\left(x, u_{n}, \nabla u_{n}\right)\right)_{n}$ is bounded in $L^{p_{i}^{\prime}(\cdot)}(\Omega)$, and Lemma 2.1, we obtain

$$
a_{i}\left(x, u_{n}, \nabla u_{n}\right) \rightarrow a_{i}(x, u, \nabla u) \quad \text { in } L^{p_{i}^{\prime}(\cdot)}(\Omega) .
$$

Now, let $E$ be a measurable subset of $\Omega$. For all $m>0$, we have by using (1.6)

$$
\begin{aligned}
& \int_{\Omega}\left|g\left(x, u_{n}, \nabla u_{n}\right)\right| \mathrm{d} x \\
\leq & h(m) \int_{E \cap\left\{\left|u_{n}\right| \leq m\right\}}\left(c(x)+\sum_{i=1}^{N}\left|D_{i} T_{m}\left(u_{n}\right)\right|^{p_{i}(x)}\right) \mathrm{d} x+\int_{E \cap\left\{\left|u_{n}\right|>m\right\}}\left|g\left(x, u_{n}, \nabla u_{n}\right)\right| \mathrm{d} x .
\end{aligned}
$$

Since $\left(D_{i} T_{m}\left(u_{n}\right)\right)_{n}$ converges strongly in $L^{p_{i}(\cdot)}(\Omega)$, then for all $\varepsilon>0$, there exists $\delta>0$ such that meas $(E)<\delta$ and

$$
h(m) \sum_{i=1}^{N} \int_{E}\left|D_{i} T_{m}\left(u_{n}\right)\right|^{p_{i}(x)} \mathrm{d} x<\frac{\varepsilon}{3} \quad \text { and } \quad h(m) \int_{E} c(x) \mathrm{d} x<\frac{\varepsilon}{3} .
$$

On the other hand, using $T_{1}\left(u_{n}-T_{m-1}\left(u_{n}\right)\right)$ as a test function in (3.1) for $m>1$, we obtain

$$
\int_{\left\{\left|u_{n}\right|>m\right\}}\left|g\left(x, u_{n}, \nabla u_{n}\right)\right| \mathrm{d} x \leq \int_{\left\{\left|u_{n}\right|>m-1\right\}}|f| \mathrm{d} x,
$$


there exists $m_{0}>0$ such that

$$
\int_{\left\{\left|u_{n}\right|>m\right\}}\left|g\left(x, u_{n}, \nabla u_{n}\right)\right| \mathrm{d} x<\frac{\varepsilon}{3} \quad \text { for all } m>m_{0} .
$$

Using (3.21) and (3.22), we deduce the equi-integrability of $g\left(x, u_{n}, \nabla u_{n}\right)$. In view of Vitali's theorem, we obtain

$$
g\left(x, u_{n}, \nabla u_{n}\right) \rightarrow g(x, u, \nabla u) \quad \text { in } L^{1}(\Omega) .
$$

Finally for, $v \in W_{0}^{1, p(x)}(\Omega) \cap L^{\infty}(\Omega)$, we have

$$
\sum_{i=1}^{N} \int_{\Omega} a_{i}\left(x, T_{n}\left(u_{n}\right), \nabla u_{n}\right) D_{i} v \mathrm{~d} x+\int_{\Omega} g\left(x, u_{n}, \nabla u_{n}\right) v \mathrm{~d} x=\int_{\Omega} T_{n}(f) v \mathrm{~d} x .
$$

Letting $n \rightarrow+\infty$, we can easily pass to the limit in this equation, to see that this last integral identity is true for $u$ instead of $u_{n}$. This proves Theorem (2.2).

Example 3.1. As a prototype example, we consider the model problem

$$
\left\{\begin{aligned}
-\sum_{i=1}^{N} D_{i}( & \left.\frac{|u|^{\frac{\bar{p}(x)}{p_{i}(x)}\left(p_{i}(x)-1\right)}\left(1+\left|D_{i} u\right|\right)^{-1} D_{i} u+\left|D_{i} u\right|^{p_{i}(x)-2} D_{i} u}{(1+|u|)^{\gamma_{i}(x)}}\right) \\
& +u \sum_{i=1}^{N}\left|D_{i} u\right|^{p_{i}(x)}=f, \text { in } \Omega, \\
u=0, & \text { in } \partial \Omega,
\end{aligned}\right.
$$

where $f \in L^{1}(\Omega)$ and $\gamma_{i}: \bar{\Omega} \rightarrow \mathbb{R}^{+}, p_{i}: \bar{\Omega} \rightarrow(1,+\infty)$ as in Theorem 2.2.

\section{Acknowledgments}

The authors would like to thank the referees for the useful comments and suggestions that substantially helped improving the quality of the paper.

\section{References}

[1] Li Z., Gao W., Existence results to a nonlinear $p(x)$ - Laplace equation with degenerate coercivity and zero-order term : renormalized and entropy solutions. Appl. Anal. 95 (2) (2015), $1-17$.

[2] Zhang X., Fu Y., Solutions for nonlinear elliptic equations with variable growth and degenerate coercivity. Annali di Matematica. 193 (1) (2014), 133-161.

[3] Ayadi H., Mokhtari F., Nonlinear anisotropic elliptic equations with variable exponents and degenerate coercivity. Electron. J. Differ. Equations. 45 (2018) (2018), 1-23. 
[4] Ayadi H., Mokhtari F., Entropy solutions for nonlinear anisotropic elliptic equations with variable exponents and degenerate coercivity. Complex Variables and Elliptic Equations. DOI: 10.1080/17476933.2019.1615899 (2019), 1-23.

[5] Mokhtari F., Anisotropic elliptic equations in $\mathbb{R}^{N}$ with variable exponents and locally integrable data. Nonlinear. Math. Methods Appl. 40 (2017), 2265-2276.

[6] Mokhtari F., Mecheter R., Anisotropic degenerate parabolic problems in $\mathbb{R}^{N}$ with variable exponent and locally integrable data. Mediterr. J. Math. 16 (3) (2019), 1-21.

[7] Mokhtari F., Regularity of the solution to nonlinear anisotropic elliptic equations with variable exponents and irregular data. Mediterr. J. Math. 14 (2017), 1-18.

[8] Naceri M., Mokhtari F., Anisotropic nonlinear elliptic systems with variable exponents and degenerate coercivity. Appl. Anal. DOI: 10.1080/00036811.2019.1682136 (2019), 1-21.

[9] Fan X., Shen J., Zhao D., Sobolev embedding theorems for spaces $W^{k, p(x)}(\Omega)$. J. Math. Anal. Appl. 262 (2001) (2001), 749-760.

[10] Fan X., Anisotropic variable exponent Sobolev spaces and $\vec{p}(x)-$ Laplacian equations. Complex Var. Elliptic Equations. 56 (7-9) (2011), 623-642.

[11] Kovacik O., Rakosnik J., On spaces $L^{p(x)}$ and $W^{1, p(x)}$. Czechoslovak Math. J. 41 (4) (1991), 592-618.

[12] Benboubker M.B., Azroul E., Barbara A., Quasilinear elliptic problems with nonstandard growths. Electron. J. Differ. Equations. 2011 (62) (2011), 1-16.

[13] Benboubker M.B., Chrayteh H., Hjiaj H., Chihab Y., Existence of solutions in the sense of distributions of anisotropic nonlinear elliptic equations with variable exponent. Topol. Methods Nonlinear Anal. 46 (2) (2015), 665-693.

[14] Lions J.L., Quelques méthodes de résolution des problèmes aux limites non linéaires. Dunod, 1969. 